\title{
GPU-based normalized cuts for road extraction using satellite imagery
}

\author{
J Senthilnath, S Sindhu and S N Omkar* \\ Department of Aerospace Engineering, Indian Institute of Science, Bangalore 560 012, India. \\ ${ }^{*}$ Corresponding author.e-mail: omkar@aero.iisc.ernet.in
}

This paper presents a GPU implementation of normalized cuts for road extraction problem using panchromatic satellite imagery. The roads have been extracted in three stages namely pre-processing, image segmentation and post-processing. Initially, the image is pre-processed to improve the tolerance by reducing the clutter (that mostly represents the buildings, vegetation, and fallow regions). The road regions are then extracted using the normalized cuts algorithm. Normalized cuts algorithm is a graph-based partitioning approach whose focus lies in extracting the global impression (perceptual grouping) of an image rather than local features. For the segmented image, post-processing is carried out using morphological operations - erosion and dilation. Finally, the road extracted image is overlaid on the original image. Here, a GPGPU (General Purpose Graphical Processing Unit) approach has been adopted to implement the same algorithm on the GPU for fast processing. A performance comparison of this proposed GPU implementation of normalized cuts algorithm with the earlier algorithm (CPU implementation) is presented. From the results, we conclude that the computational improvement in terms of time as the size of image increases for the proposed GPU implementation of normalized cuts. Also, a qualitative and quantitative assessment of the segmentation results has been projected.

\section{Introduction}

Roads are among the most important topographical objects for extraction from aerial and satellite imagery for use in data acquisition and update of the Geographic Information Systems (GIS). Automatic extraction of roads has been an active area of research from the past two decades now (Baumgartner et al. 1999; Valero et al. 2010). The road network information is of utmost importance for transportation, urban planning, automated road navigation, and emergency response applications. The automatic extraction of the roads is posed with many challenges. The most important ones include huge objects that might cause occlusions like shadows on the roads, the presence of vehicles, and road markings (Senthilnath et al. 2009). Therefore, we employ an algorithm that considers low level coherence of brightness, colour, and texture of the image for the automation of this semantic task (Shi and Malik 2000; Senthilnath et al. 2013). This study focuses on the extraction of roads using satellite imagery.

Several methods have been developed for road extraction and are summarized (Mayer et al. 2006). To deal with satellite images of urban and suburban areas, very few algorithms retain their reliability owing to the highly complex structure found in urban scenes (Grote et al. 2007). Semi-automated approaches require human intervention and user provided clues for the identification of features on the image. Gruen and Li (1997) formulated the problem using an active contour model in a least square context (LSB-Snake) where, by initializing

Keywords. Road extraction; normalized cuts; graphical processing unit. 
seed points, road segments are generated by optimizing an energy function based on geometric characteristics. Geman and Jedynak (1996) proposed a semi-automatic method, where, given a start point and a start direction, a road is extracted from panchromatic SPOT satellite image by playing 'tests' about the 'true hypothesis' Park and Kim (2001) have successfully applied road extraction based on template matching.

Barzohar and Cooper (1996) successfully automated the road extraction model to extract roads through dynamic programming. In their study, roads are found by Maximum Aposteriori Probability (MAP) estimation, which is handled by partitioning an image into multiple regions. In their study, road segments are extracted using the geometricstochastic model. The geometric-stochastic model of a road is built on certain assumptions in which, the width of the road is assumed to be less varying whereas abrupt change in its direction is not considered. Also, roads are assumed to have high contrast against their background and are unlikely to be short. However, these assumptions will seldom be universally applicable as road images may vary with ground resolution, road type, and density of surrounding objects (Bong et al. 2009; Youn and Bethel 2004), extracted road networks, by assuming roads are semi-regular grid pattern. This constraint may not be suitable for many European urban areas (Grote et al. 2007). Roads in different parts of the world exhibit different characteristics. The notion of straightness is more pronounced in a road in Australia than in central Europe. Similarly in India, the spectral information, textures, width of the roads, road markings, and clutter vary depending on the location (Senthilnath et al. 2009). Hence, it is helpful to include both local and global features in an extraction strategy (Grote and Heipke 2008).

From the above works, we deduce that image segmentation formulated on specific types of image scenes and road models must not only consider spatial and spectral properties but must also include global features of the image for reliable segmentation. In the present study, we have adopted the normalized cuts algorithm. It includes several spectral features to describe pixel similarity. The similarity factor takes into account several features of the image including the context information, which is necessary for the correct segmentation of images with complex entities such as built-up areas (Grote et al. 2007). In literature, normalized cut method has been successfully applied for road extraction using high resolution satellite image (Grote et al. 2007; Grote and Heipke 2008; Senthilnath et al. 2009). In Senthilnath et al. (2009), it has been observed that normalized cut method performs better than texture progressive analysis for high resolution satellite. For high resolution data, high variability leads to predominant edges where normalized cut algorithm identifies these edges to form and find the optimal 'cut' for iterative segmentation of the image. Hence, the use of high resolution image stimulates a better percentage of correctness using normalized cut algorithm in comparison with conventional methods.

Normalized cut is a grouping algorithm based on the view that perceptual grouping should be a process that aims to extract local and global impressions of a scene (Shi and Malik 2000). The drawback of this method is that the calculation is computationally expensive and as the image size grows, the images need to be split into subsets for processing (Grote et al. 2007). With the increase in image size, the image segmentation time also increases. An insight into fast processing based on the power of GPU (Graphical Processing Unit) plays an important role at this juncture. Hence, in our study we use GPU implementation of normalized cuts for road extraction problem using high resolution satellite image to increase the computation time.

At the inception of NVIDIA's CUDA (Compute Unified Device Architecture) - a hardware and software co-processing architecture for parallel computing, several image processing algorithms have been implemented using GPGPU (Moreland and Angel 2003; Rubin et al. 2011; Wendykier and Nagy 2011). Recently, to increase the computational speed, GPU is widely used. GPU has evolved from special-purpose graphics rendering processor to a programmable processor, while ease of programmability has been the most important feature (Yang et al. 2008). Since the real-time aspect is getting more and more important in image processing and especially in image segmentation, parallel hardware architectures and programming models for multi-core computing have been developed to achieve acceleration (Abramov et al. 2011). In Pan et al. (2008), medical image segmentation has been performed using the watershed method and region growing algorithm on CUDA enabled GPUs. Abramov et al. (2011) have achieved image segmentation by super paramagnetic clustering using metropolis algorithm. This work has been executed on the framework NVIDIA CUDA. Apart from the above works, Moreland and Angel (2003) and Yang et al. (2008) reflect many other image processing algorithms - the FFT and histogram equalization respectively, that have been adapted to run on the GPU.

To implement the normalized cuts algorithm on the GPU, we have made use of a middleware called Jacket (Acclereyes $\left.{ }^{\circledR}\right)$. Jacket enables us to execute the computationally expensive parts of the algorithm on the GPU with a negligible change in its 
counterpart implementation (Rubin et al. 2011). It provides a transparent mapping by allowing the standard MATLAB code to be run on the GPU. In Wendykier and Nagy (2011), 2D and 3D image deblurring techniques have been implemented on the GPU using the Jacket software - the results show a significant acceleration in time. In addition, works in image processing, implemented on Jacket, that have proven to produce convincing results can be found in Rubin et al. (2011) and Kong et al. (2010).

In this paper, we consider a CPU implementation of normalized cuts method that was used in earlier studies for the image segmentation (Shi and Malik 2000). Previous works on road extraction using normalized cuts are available in Senthilnath et al. (2009) and Grote et al. (2007). These works have successfully implemented normalized cuts algorithm to extract road segments in urban and suburban scenes but have faced the problem of increasing time taken for the segmentation of larger images. In this paper, we present a GPUbased implementation of normalized cuts using Jacket software for the road extraction problem. Our paper addresses the issue of road extraction considering multi-sensor panchromatic satellite images with different scenarios such as Indian and Australian. The roads have been extracted in three stages namely pre-processing, image segmentation, and post-processing. Initially, the image is preprocessed to improve the tolerance by reducing the clutter (that mostly represents the buildings, vegetation, and fallow regions). Further, the road segments are extracted using the normalized cuts algorithm. For the road segmented image, erosion and dilation have been applied as postprocessing and then the road extracted image is overlaid on the original image. The image segmentation mainly depends on the spectral sensitivity and spatial resolution of the sensor. We have made use of high-resolution panchromatic satellite images such as IKONOS and QuickBird.

The important aspects of the present work are: (a) implementation of the normalized cut method on the GPU to achieve acceleration; (b) the acceleration in time is compared between CPU and GPU implementation of normalized cuts for road extraction using satellite imagery (IKONOS and QuickBird); (c) a quantitative and qualitative analysis of the road segmented results is done considering the results of both the CPU and GPU-based normalized cuts. From the results, we conclude that the computational improvement in terms of time as the size of image increases for the proposed GPU implementation of normalized cuts.

The remainder of this paper is organized as follows: section 2 describes the methodology for road extraction, section 3 discusses system architecture and implementation, section 4 provides an overview of the study area and the results, and conclusions are presented in section 5 .

\section{Methodology}

The proposed road extraction has been broadly divided into three steps: pre-processing, application of normalized cuts algorithm on the preprocessed image, and post-processing.

\subsection{Pre-processing}

Pre-processing is required to improve the image quality and to generate the elongated road network for further processing. This involves three steps, viz., classification, grouping, and filtering.

\subsubsection{Classification}

Initially all the images were subjected to classification using the ISODATA - unsupervised classification method through ERDAS tool $\mathbb{R}$. For each image a level one classification was carried out based on two classes: roads and nonroads. Totally 15 clusters were generated among which five clusters correspond to road networks. Here, a lot of misclassifications were noted among roads and barren land. A few cases of misclassification of roads to vegetation and built-up region were also seen.

\subsubsection{Grouping}

The purpose of grouping comes into picture postclassification due to the occurrence of misclassification. A nearest neighbourhood grouping (NNG) operation is applied to the above-classified image. This smoothes the spectral response within the pixel's local neighbourhood. In this process the vehicular occlusions and small patches of road contrast abnormalities are eliminated. In this method, a pixel is chosen and its surrounding eight neighbouring pixels' classes are considered. Now, the class of the considered pixel is assigned by majority vote of similar class. The pixel retains its class otherwise. Road pixels in the neighbourhood grow over such noise elements and homogeneous regions/segments are generated.

\subsubsection{Filtering}

The method of road extraction in urban areas poses a challenge because of the spectral reflectance of some of the old buildings (or buildings with a type of construction that renders a dark road like effect) which resembles the road surface. Such buildings form the clutter; hence these nonroad structures 
need to be removed. Median filtering technique can be applied as it is very useful in removing random noise (Huang et al. 1979). The median filtering approach is similar to averaging filter, where output pixel is set to an average of the pixel values considering the neighbourhood of the corresponding input pixel. However, in median filtering, the value of an output pixel is determined by the median of the neighbourhood pixels. The median is much less sensitive than the mean to extreme values (called outliers) (Huang et al. 1979). Median filtering is therefore better suited to remove these outliers without reducing the sharpness of the image.

\subsection{Image segmentation using normalized cuts}

In this section, we pursue image segmentation in the framework of normalized cuts introduced by Shi and Malik (2000). For the pre-processed image, road segments are extracted automatically using normalized cuts.

Inspired by spectral graph theory (Chung 1997), Shi and Malik (2000) formulated visual grouping as a graph partitioning problem. Normalized cut is a graph-based method which is used to divide an undirected graph with weighted edges into segments with similar features. The method in detail is described in Shi and Malik (2000). In image segmentation, pixels are the entities that we want to partition and hence become the nodes of the graph. The edges between two nodes indicate the strength with which these two nodes belong to one group. The image is considered to be a weighted graph where the nodes $i$ and $j$ are pixels and edge weights, $W_{i j}$, denote a local measure of similarity between the two pixels. The criterion for partitioning the graph is to minimize the sum of weights of connections across the groups and maximize the sum of weights of connections within the groups (Shi and Malik 2000).

Theoretically, every pixel can be connected to every other pixel in the graph. But, in practice only pixels in the vicinity (by specifying the region of neighbourhood parameter) of the reference pixel are connected with weights different from zero (Grote et al. 2007).

Consider an undirected graph $G=(V, E)$, where $V$ is the set of nodes and $E$ is the set of edges. A pair of nodes $p$ and $q$ is connected by an edge and is weighed by $W(p, q)$. Let $X$ and $Y$ be a partition of the graph where $X \cup Y=V$ and $X \cap Y=\phi$. Therefore cost of cut is defined as the similarity between the groups $X$ and $Y$ and it is formulated as:

$$
\operatorname{Cut}(X, Y)=\sum_{p \in X, q \in Y} w(p, q)
$$

Minimum cut is the cut of minimum weight, where weight of cut $(X, Y)$ is given in equation (1). The limitation of using minimum cut is described in Shi and Malik (2000). However, the minimum cut criterion favours grouping small sets of isolated nodes in the graph because it cuts with lesser weight than the ideal cut (Shi and Malik 2000). In other words, the minimum cut usually yields overclustered results when it is recursively applied. This calls for a different cut criterion. Shi and Malik (2000) proposed to use a normalized cut criterion

$$
N \text { cut }(X, Y)=\frac{\operatorname{cut}(X, Y)}{\operatorname{volume}(X)}+\frac{\operatorname{cut}(X, Y)}{\operatorname{volume}(Y)}
$$

where, volume $(X)$ is the sum of cost of all edges that touch $X$ and volume $(Y)$ is the sum of cost of all edges that touch $Y$, i.e.,

$$
\begin{aligned}
& \text { volume }(X)=\operatorname{assoc}(X, V)=\sum_{p \in X, q \in V} w(p, q) \\
& \text { volume }(Y)=\operatorname{assoc}(Y, V)=\sum_{p \in Y, q \in V} w(p, q)
\end{aligned}
$$

where $\operatorname{assoc}(X, V)$ is the total connection from nodes in $X$ to all the nodes in the graph and $\operatorname{assoc}(Y, V)$ is the total connection from nodes in $Y$ to all the nodes in the graph.

One prominent advantage of this algorithm is that it takes into account both the local and global characteristics of the image. Local characteristics are based on image cues like intensity and texture that are incorporated into the similarity matrix by the calculation of weights between neighbouring pixels. The global characteristics are involved in the calculation of global optimal partition criterion (Grote et al. 2007). The global optimal partition criterion is shown in equation (5). Let $W$ be the cost matrix, i.e., $W(i, j)=c_{i, j}$; is the weight between the nodes $i$ and $j$ in the graph and $D$ be the diagonal matrix such that $D(i, i)$ is the sum of costs from node $i$ to all the other nodes in the graph and $D(i, j)=0$. Based on this input, Shi and Malik (2000) showed that the optimal partition can be found by computing:

$$
\min N \operatorname{cut}(X, Y)=\min _{y} \frac{y^{T}(D-W) y}{y^{T} D y}
$$

Such that $y(i) \in\{1,-b\}, 0<b \leq 1$, and $y^{T} D 1=0$.

A slight relaxation induced in terms of $y(i)$ being able to take up real values would lead to the minimization of equation (5). Equation (5) can now be solved as a generalized eigen value problem.

$$
(D-W) y=\lambda D y \text {. }
$$


Hence, the global minimum criterion that is to be met is computed efficiently. This has a significant advantage where small disturbances like short or weak edges are ignored by the algorithm (Grote et al. 2007).

In normalized cut method for extracting road segments, each individual edge weight is a measure of the similarity between two connected pixels. The similarity measure is basically application dependent. For example, if there is a significant variation in the intensities between road and nonroad regions, then calculation of the similarity matrix is carried out based on intensity. However, it is also possible to combine various similarity criteria based on the image cues (Senthilnath et al. 2009). The weight, which is the combination of all similarity measures, has a value between 0 and 1 . The graph representing the image is then cut into segments aiming at a large dissimilarity between different segments and at the same time a large similarity within the segments. The weight calculated for each pixel pair is then inserted into a symmetrical matrix the size of whose rows and columns is each equal to the number of pixels in the image.

$W(i, j)=$ $\begin{cases}e^{-\left\|F_{i}-F_{j}\right\| / \sigma_{I}^{2}} \times e^{-\left\|X_{i}-X_{j}\right\| / \sigma_{X}^{2}} & \text { if }\left\|X_{i}-X_{j}\right\|_{2}<r \\ 0 & \text { otherwise }\end{cases}$

where $\left\|F_{i}-F_{j}\right\|=$ image feature similarity, $\left\|X_{i}-X_{j}\right\|=$ spatial proximity and $r=$ region of neighbourhood definition.

With the image cases that we have worked on, the basis for calculation of similarity matrix is the intensity factor. Once the similarity matrix is formed, the minimization of the Ncut parameter (indicated in equation 5) is done, the number of partitions possible or in essence, the number of eigen vectors obtained after solving the generalized eigen problem is equivalent to the number of pixels (Shi and Malik 2000).

\subsection{Post-processing}

As a part of post-processing, the extracted road region obtained using normalized cuts method is converted into binary image format. The morphological operations such as erosion and dilation are used to remove the noise obtained after segmentation (Haralick et al. 1987). Erosion removes unwanted pixels on object boundaries. Dilation adds pixels to the boundaries of objects in an image. It generally increases the sizes of objects, by filling in holes, broken areas, and also connecting areas that are separated by spaces. In order to illustrate the accuracy, this binary image is overlaid on the original panchromatic image. This leads to display the road topology by avoiding the complex noise elements. In the overlaid imazge, the thin lines indicate the road topology.

\subsection{Panchromatic satellite image}

One of the study areas chosen is the Hobart city in Tasmania, Australia. We have used IKONOS panchromatic image with a resolution of $1 \mathrm{~m}$. The size of the IKONOS image used for this study is $624 \times 488$ pixels.

The second study area is the urban part of the Bangalore city in Karnataka, India. We use an image obtained from the QuickBird high-resolution satellite owned and operated by Digital Globe. QuickBird's panchromatic image has a resolution of $0.61 \mathrm{~m}$. The size of the image is $900 \times 900$ pixels.

\subsection{Performance evaluation}

We have adopted three measures from the work of Wiedemann et al. (1998). In Wiedemann et al. (1998), the matched extracted data are denoted as true positive with length $T P$ emphasizing the fact that the extraction algorithm has indeed found a road, the unmatched extracted data is denoted as false positive with length $F P$, because the extracted road hypotheses are incorrect. The unmatched reference data are denoted as false negative with length $F N$. The quality measures used to evaluate the reference data and automatic road extraction data are:

- Completeness: This is the percentage of the reference data which is explained by the extracted data, i.e., the part of the reference network which lies within the buffer around the extracted data. The optimum value for completeness is 1 (or $100 \%$ efficiency).

$$
\begin{aligned}
\text { Completeness } & =\frac{\text { length of matched reference }}{\text { length of reference }} \\
& =\frac{T P}{T P+F N}
\end{aligned}
$$

- Correctness: It represents the percentage of correctly extracted road data, i.e., the part of the extracted data which lies within the buffer around the reference network. The optimum value for correctness is 1 (or 100\% efficiency).

$$
\begin{aligned}
\text { Correctness } & =\frac{\text { length of matched extraction }}{\text { length of extraction }} \\
& =\frac{T P}{T P+F P}
\end{aligned}
$$


- Quality: It is a measure of the goodness of the final result by combining both completeness and correctness into a single measure. The optimum value for quality is 1 (or $100 \%$ efficiency).

$$
\begin{aligned}
\text { Quality } & =\frac{\text { length of matched extraction }}{\begin{array}{c}
\text { length of extraction+length } \\
\text { of unmatched reference }
\end{array}} \\
= & \frac{T P}{T P+F P+F N}
\end{aligned}
$$

\section{System architecture and implementation}

For the algorithm implementation, we have made use of a quad core CPU-Q9400 running at 2.66 $\mathrm{GHz}$ and with $3.99 \mathrm{~GB}$ of RAM. The configuration of the GPU is NVIDIA GeForce GTX 460 SE with $1024 \mathrm{MB}$ of memory. In this section, the system architecture for satellite image processing, and CPU and GPU implementation of normalized cuts algorithm has been discussed.

\subsection{System architecture}

Acclereyes ${ }^{\circledR}$ Jacket is a transparent middleware basically built on the NVIDIA CUDA technology. Jacket basically relies on a set of dubbed ' $g$ ' prefixed classes which mimic the native Matlab classes by performing the same function on the

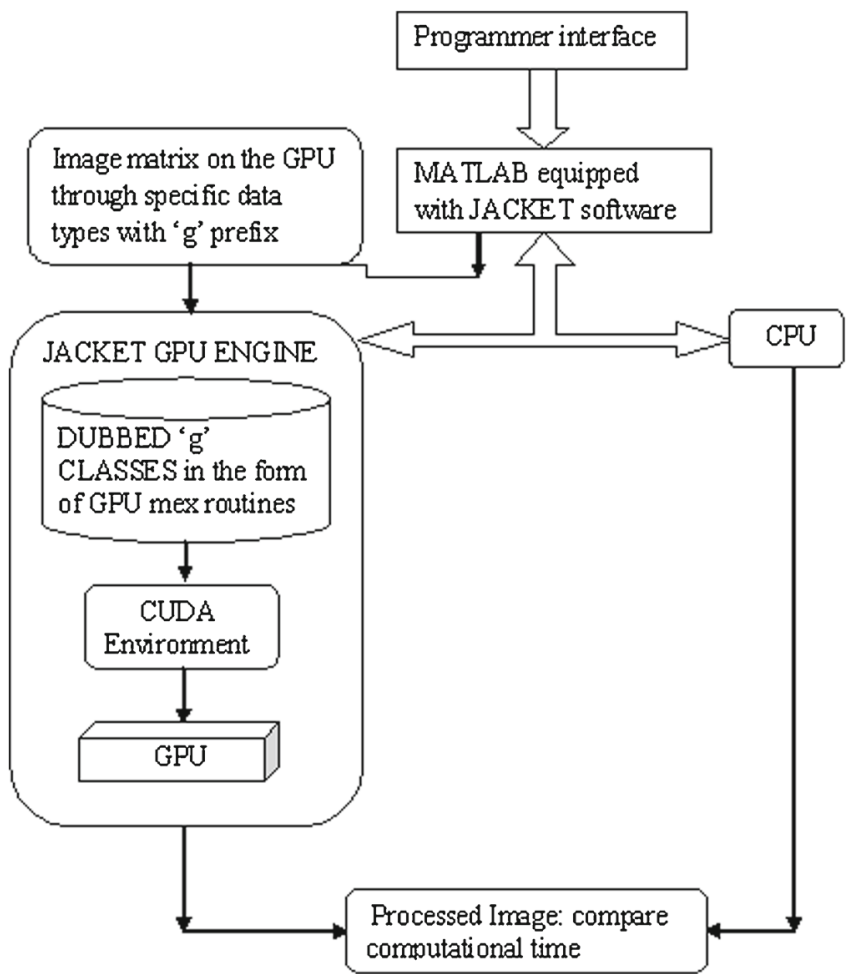

Figure 1. Jacket middleware architecture.
GPU. A pictorial representation of Jacket architecture is shown in figure 1 . These classes overload the built-in set of base Matlab functions which provide full GPU computation functionality in the Matlab environment through polymorphism (Acclereyes 2011). In essence, the standard functions present on Matlab are made available to the ' $g$ ' objects via GPU-enabled mex routines. These functions perform the same counterpart Matlab functions faster. Jacket uses some special features to execute the dubbed classes on GPU. Some of them are lazy execution, just in time compilation (JIT), garbage collection, thread extraction, and instruction caching (Acclereyes 2011).

\subsection{CPU implementation of normalized cuts}

We first explain the CPU implementation of the algorithm and next the changes incorporated to convert it to a GPU implementation.

The flowchart of the algorithm is presented in figure 2. The image matrix, $I$ of size $X \times Y$ (where, $X$ - number of rows and $Y$ - number of columns) is given as the input to the algorithm. The parameters $\sigma_{x}$ and $\sigma_{I}$ have been tuned based on the considered image. ' $R$ ' indicates the region of neighbourhood definition and ' $N$ cut' indicates a threshold for repetitive partitioning - all these parameters are user specified.

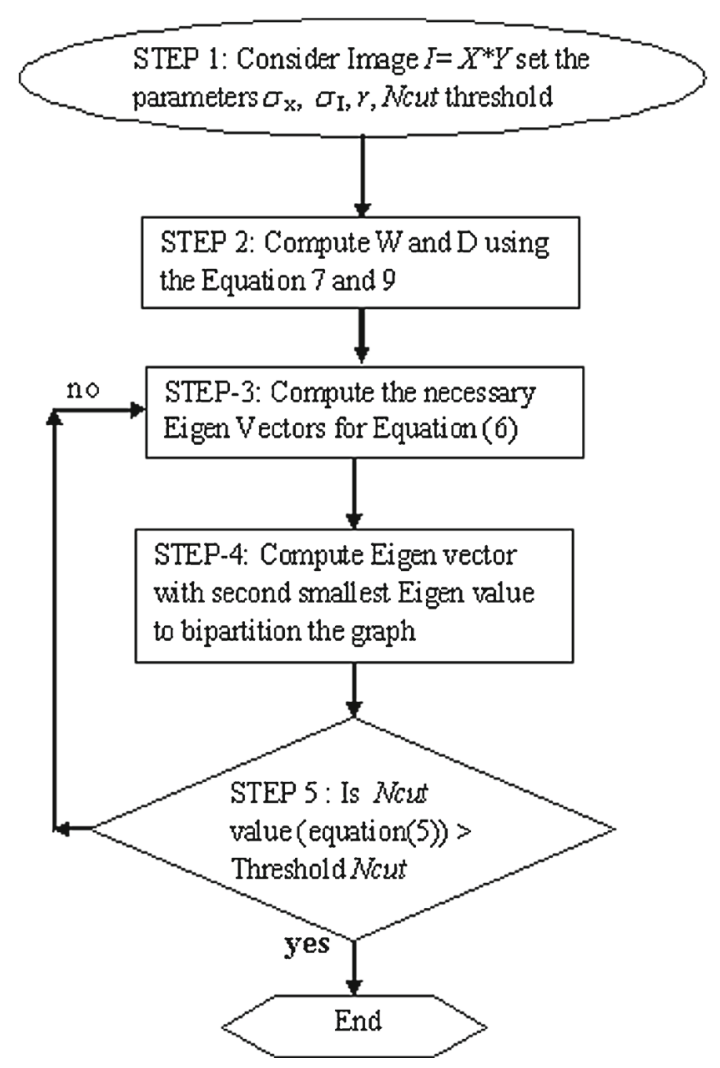

Figure 2. CPU implementation of normalized cuts algorithm. 


\subsection{GPU implementation of normalized cuts}

GPU implementation requires two important classes of modifications - the code vectorization and then the GPU execution.

In Matlab, 'vectorization' refers to taking advantage of polymorphism, a compiler feature that allows the same line of code to apply to scalars, vectors, or matrices. Matlab can perform these vectorized calculations much more efficiently than loops and automatically multithread some operations (Rubin et al. 2011). We have used the CPUbased normalized cuts implementation from the earlier study of Seo (2006). The same implementation has been modified for GPU-based normalized cuts. The flowchart shown in figure 3 details the steps involved in the GPU implementation. The steps that have undergone modifications have been explained below:

Step 1: Given an image $I$ of size $X \times Y$, in order to carry out further calculations on the GPU (using Jacket software), we cast the main image variable onto GPU, by the following syntax

$$
\begin{aligned}
I= & \text { imread('image.jpg'); // on CPU: where } I \\
& \text { is of 'single 'data type.// } \\
I= & \text { gsingle }(I) ; / / \text { on GPU through specific } \\
& \text { data type of single precision by the } \\
& \text { prefix ' } g \text { '// }
\end{aligned}
$$

\begin{tabular}{|c|c|c|c|c|c|c|}
\hline \multirow[b]{2}{*}{ Images } & \multicolumn{2}{|c|}{ Completeness } & \multicolumn{2}{|c|}{ Correctness } & \multicolumn{2}{|c|}{ Quality } \\
\hline & $\overline{\mathrm{CPU}}(\%)$ & GPU (\%) & $\overline{\mathrm{CPU}}(\%)$ & GPU (\%) & $\overline{\mathrm{CPU}}(\%)$ & GPU $(\%)$ \\
\hline $159 \times 642$ & 82.5 & 77.5 & 81 & 75.3 & 66.5 & 57.5 \\
\hline $327 \times 467$ & 87.2 & 84.4 & 100 & 92.4 & 85.2 & 76.3 \\
\hline $624 \times 488$ & 88.4 & 88.6 & 90.3 & 89.8 & 79.5 & 79.3 \\
\hline $900 \times 900$ & 95.3 & 99.5 & 94.9 & 91.8 & 90.5 & 91.4 \\
\hline
\end{tabular}

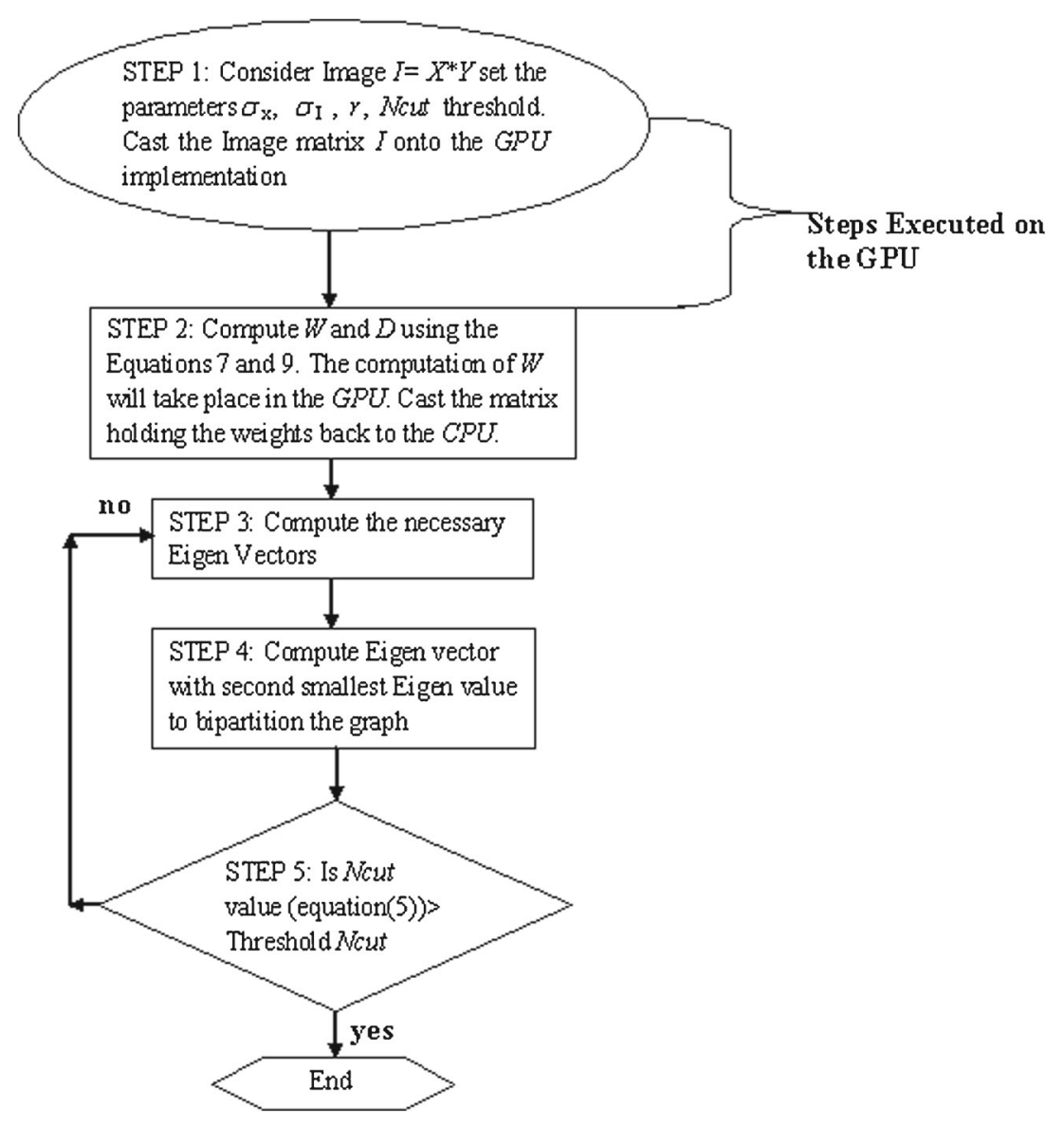

Figure 3. GPU implementation of normalized cuts algorithm.

Table 1. Performance evaluation results. 
Step 2: One among the more time consuming parts of the algorithm is the similarity matrix computation. The acceleration in terms of time is achieved basically by performing every iteration of the matrix-vector multiplication on the GPU.

The similarity matrix $(W)$ computation involves $N \times N$ iterations where $N=X \times Y$. Thus by performing these multiplications on the GPU we have achieved a 'per-iteration-speedup'. The $W$ calculated is stored as a full matrix since sparse matrices are not fully supported by Jacket. Another computationally expensive part of this algorithm happens to be the evaluation of selective eigen vectors and eigen values. Jacket does not support the associated package, viz., LAPACK routine (Wendykier and Nagy 2011).

Let

$$
\text { Full matrix }=W_{1} \text {. }
$$

The variable is transferred back to the CPU by merely changing its data type

$$
W=\operatorname{single}\left(W_{1}\right) .
$$

As a result, after the similarity matrix has been calculated, the full matrix is converted to a sparse matrix and further calculations are as per the CPU implementation.

\section{Results and discussion}

In this section, we present the results obtained for the problem of road extraction using panchromatic satellite image. In our study, we consider two panchromatic satellite images. The first satellite image - IKONOS image is the Hobart city in Tasmania, Australia. The second satellite image - QuickBird image is the Bangalore city in Karnataka, India.

The aim of this study is to present the results obtained for IKONOS and QuickBird panchromatic images for road extraction using normalized cuts - CPU and GPU implementation. Finally, the performance of the road extraction and processing time as the increase in the size of satellite image is evaluated and analyzed.

The first two images shown in table 1 are smaller parts of the $624 \times 488$ image. The increasing sizes of the images are considered to project the computational improvement in time. For illustrating the result, we consider the extracted road image $(624 \times 488$ pixels $)$ using CPU and GPU implementation of normalized cuts as shown in figures 4 and 5 , respectively. The segmented QuickBird images of size $900 \times 900$ pixels using CPU and GPU are shown in figures 6 and 7 , respectively.
In this study, normalized cut algorithm uses intensity factor as similarity measures. The panchromatic images are segmented into two segments: roads and nonroads. This is done by using normalized cut algorithm with spectral reflectance of each pixel. For this segmented image, morphological operation is applied to remove other regions falsely segmented as roads. In this process, other urban areas such as paved parking areas and nonroad regions are also segmented as roads. This is because the paved parking areas and nonroad regions have spectral reflectance similar to roads. The other urban areas such as paved parking areas and nonroad regions are called false roads. In figures $4-7$, we can observe false roads are extracted as road segment.

Evaluation of road extraction system is done by comparing the results with the reference data. The quality measures used for road extraction using normalized cut method are completeness, correctness, and quality factor. Table 1 shows the numeric results of the qualitative analysis. The average completeness is $88.35 \%$ for CPU-based results and $87.5 \%$ for GPU-based results. The average correctness is around $91.6 \%$ and $87.3 \%$ for $\mathrm{CPU}$ and GPU-based implementations, respectively. Average quality factor is $80.4 \%$ on CPU results and $76.1 \%$ on GPU-based results.

Road segmentation involves fine tuning of the four parameters involved in normalized cut. After

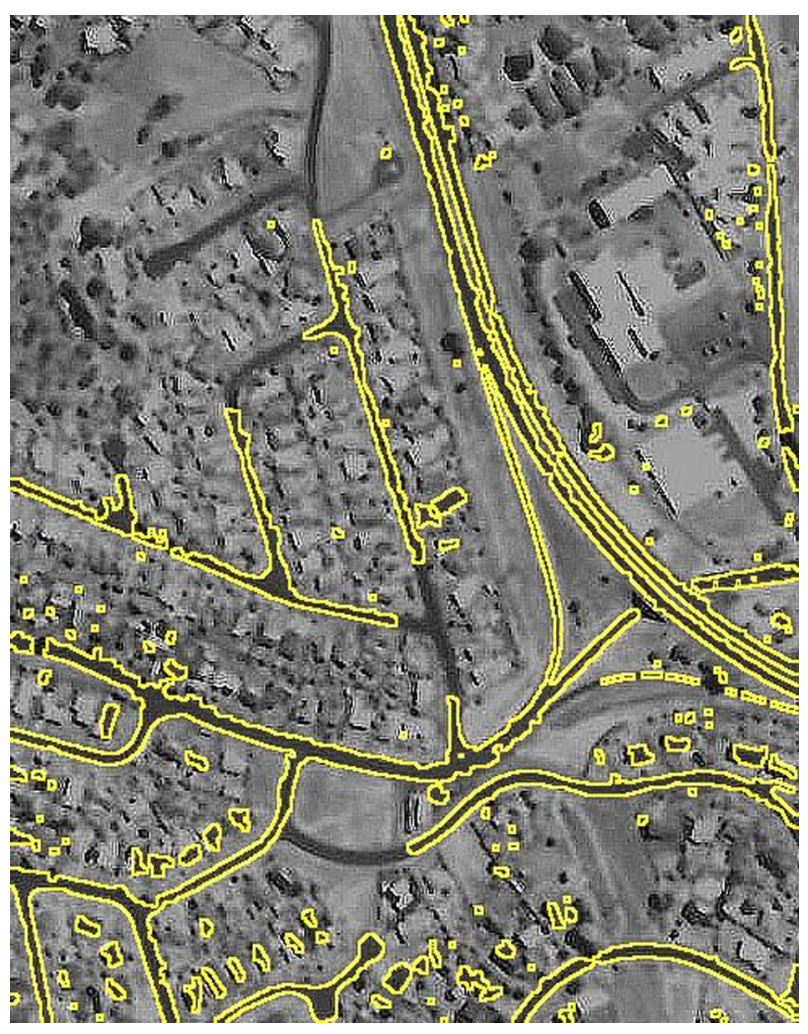

Figure 4. CPU segmented IKONOS image $(624 \times 488)$. 


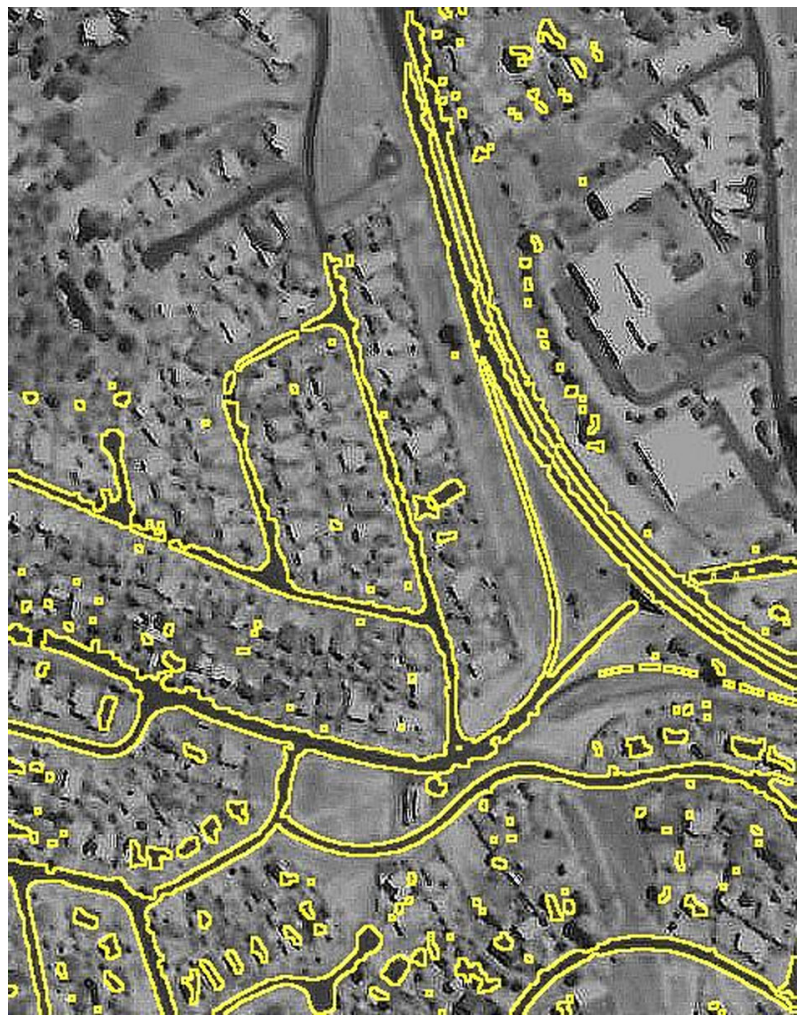

Figure 5. GPU segmented IKONOS image $(624 \times 488)$.

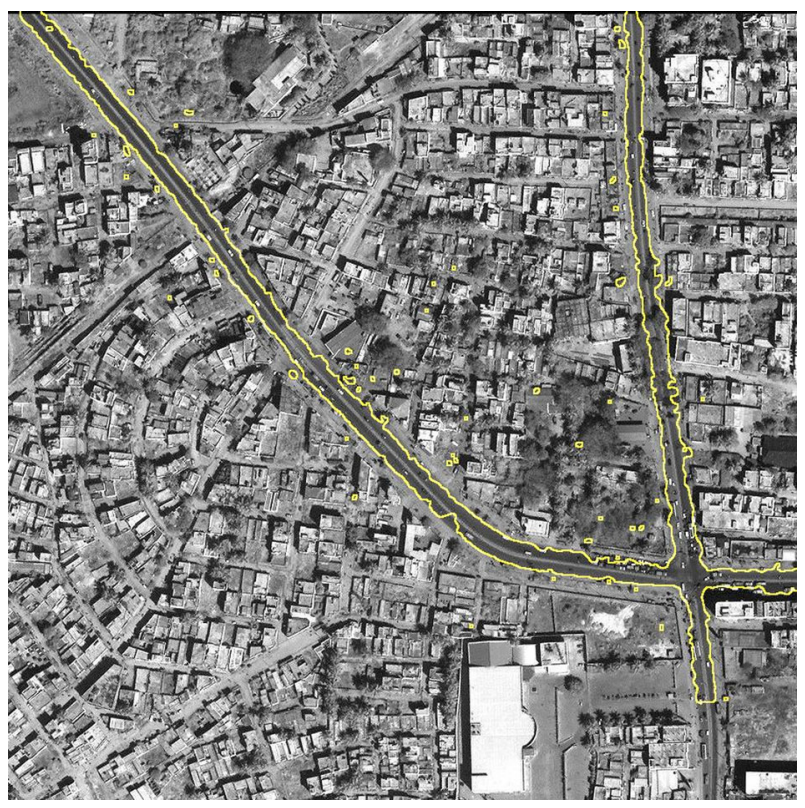

Figure 6. CPU segmented QuickBird image $(900 \times 900)$.

several runs, these four parameter values of normalized cut algorithm on CPU and GPU were empirically selected for our experiments. For all our images, the parameters are set to values as shown in table 2. The effect of increasing the ' $R$ ' parameter is that the algorithm yields larger segments. The $\sigma_{x}$ and $\sigma_{I}$ have to be tuned carefully to obtain meaningful road segmentation. The values of $\sigma_{x}$

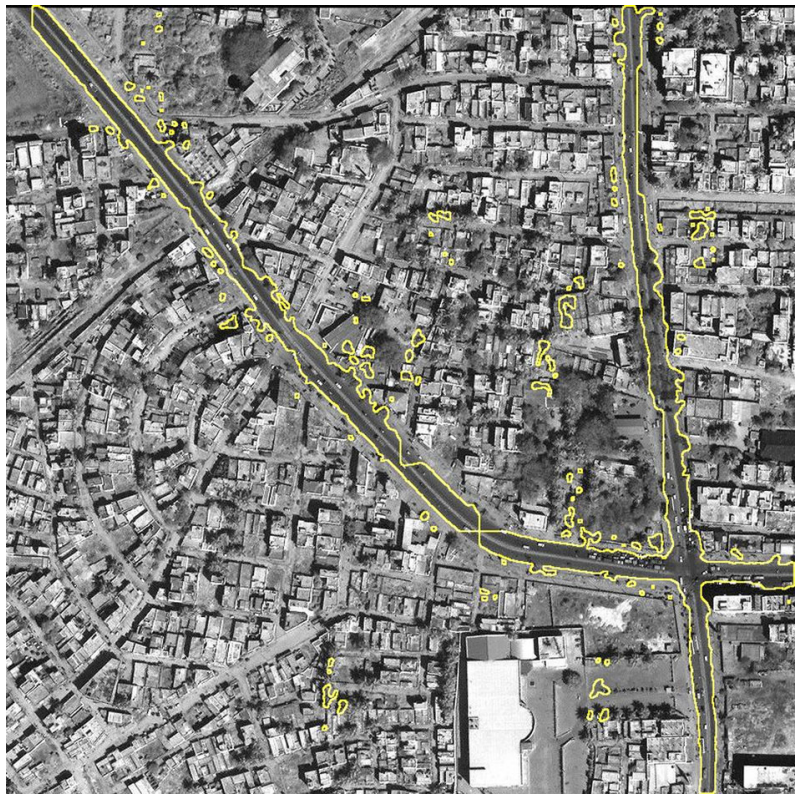

Figure 7. GPU segmented QuickBird image $(900 \times 900)$.

and $\sigma_{I}$ are carefully tuned for each image considered for our experiment. Typical values are set in the range of $10-20 \%$ of the range of edge intensity captured in each image under study. A qualitative comparison of table 1 and table 2 - an important observation is that the value of $R$ is set optimally and as it increases in delta steps we can obtain larger segments of roads from the image. However, to look at an ideal case from our result, consider the $900 \times 900$ image where the value of $R$ is 1.4 in CPU implementation and 1.69 in GPU. Also, it was observed that Ncut parameter determines the total number of segments formed in the output. The false roads extracted in figures $4-7$, are as a result of a lower Ncut threshold value. To minimize the presence of false roads, an increase in the threshold Ncut value was attempted, but the observation was that we would sometimes not obtain even the accurate segmentation of the feature, i.e., roads and the performance of completeness decreases. The completeness and the quality are promising in the case of GPU while the correctness is slightly affected and goes down by $3.1 \%$.

The speed-up of the algorithm can be defined as the CPU time taken divided by the time taken on the GPU for the execution of the algorithm. In the image cases that are considered, the maximum speed up has been obtained for a size of 900 $\times 900$ pixels, where the speed up is $3.16 \mathrm{X}$. From the results in table 3 , we can infer that the speedup increases with the size of the image. A plot of the image size (on the $\mathrm{x}$-axis) vs. time in seconds (on the y-axis) is shown in figure 8. Another distinct observation is the steady improvement in 
Table 2. Parameters' setting for normalized cut.

\begin{tabular}{|c|c|c|c|c|c|c|c|c|}
\hline \multirow[b]{2}{*}{ Images } & \multicolumn{4}{|c|}{ Parameter setting on $\mathrm{CPU}$} & \multicolumn{4}{|c|}{ Parameter setting on GPU } \\
\hline & $\overline{\sigma_{x}}$ & $\sigma_{y}$ & $R$ & Ncut (threshold) & $\overline{\sigma_{x}}$ & $\sigma_{y}$ & $R$ & Ncut (threshold) \\
\hline $159 \times 642$ & 8 & 2 & 1.6 & 0.01 & 9.6 & 4 & 1.5 & 0.01 \\
\hline $327 \times 467$ & 6 & 3 & 1.07 & 0.89 & 11 & 4.8 & 1.3 & 0.89 \\
\hline $624 \times 488$ & 9 & 6 & 1.5 & 0.04 & 12 & 6 & 1.4 & 0.04 \\
\hline $900 \times 900$ & 9.1 & 4 & 1.4 & 0.04 & 14 & 2 & 1.69 & 0.04 \\
\hline
\end{tabular}

Table 3. Average execution times for normalized cut implemented on MATLAB (CPU version) and on MATLAB + Jacket (GPU version).

\begin{tabular}{lccrc}
\hline Sl. no. & $\begin{array}{c}\text { Image } \\
\text { size }\end{array}$ & $\begin{array}{c}\text { CPU time } \\
(\mathrm{sec})\end{array}$ & $\begin{array}{c}\text { GPU time } \\
(\mathrm{sec})\end{array}$ & Speed-up \\
\hline 1 & $159 \times 642$ & 93.83 & 81.76 & $1.15 \mathrm{X}$ \\
2 & $327 \times 467$ & 115.84 & 76.79 & $1.51 \mathrm{X}$ \\
3 & $624 \times 488$ & 521.72 & 255.72 & $2.04 \mathrm{X}$ \\
4 & $900 \times 900$ & 3548.1 & 1122.87 & $3.16 \mathrm{X}$ \\
\hline
\end{tabular}

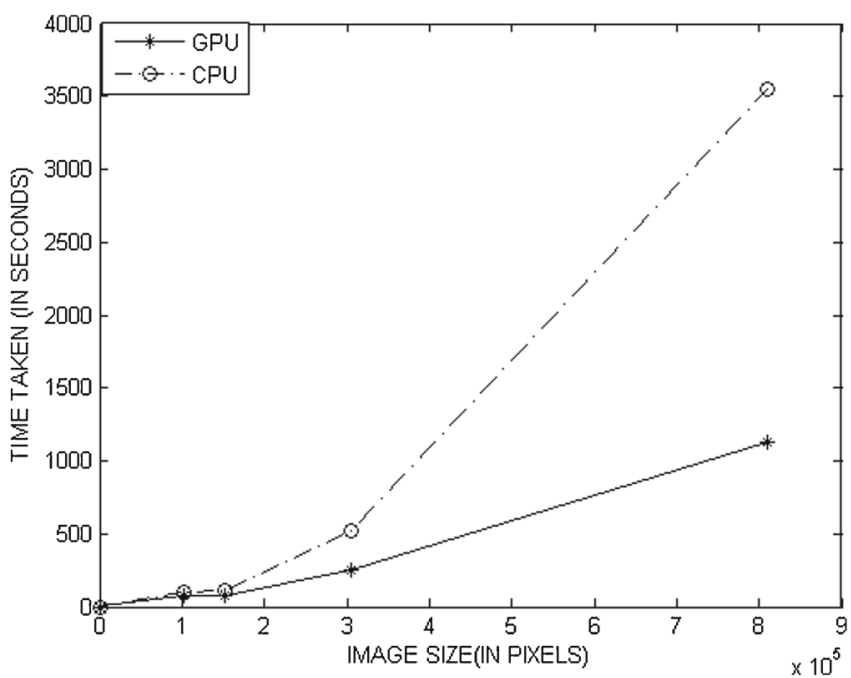

Figure 8. Image size vs. time taken to execute normalized cuts algorithm.

the quality factor with image size of GPU-based segmentation, as shown in figure 9.

The results have been recorded as an average of 10 runs of the algorithm for each image (Wendykier and Nagy 2011). The MATLAB + Jacket based results include the time taken for data transfer to and from the GPU. The timings are shown in table 3 .

\section{Conclusions}

In this work, we have demonstrated the application of normalized cuts algorithm for road extraction and implemented the normalized cuts algorithm on the GPU using Acclereyes $\mathbb{R}$. Jacket software.

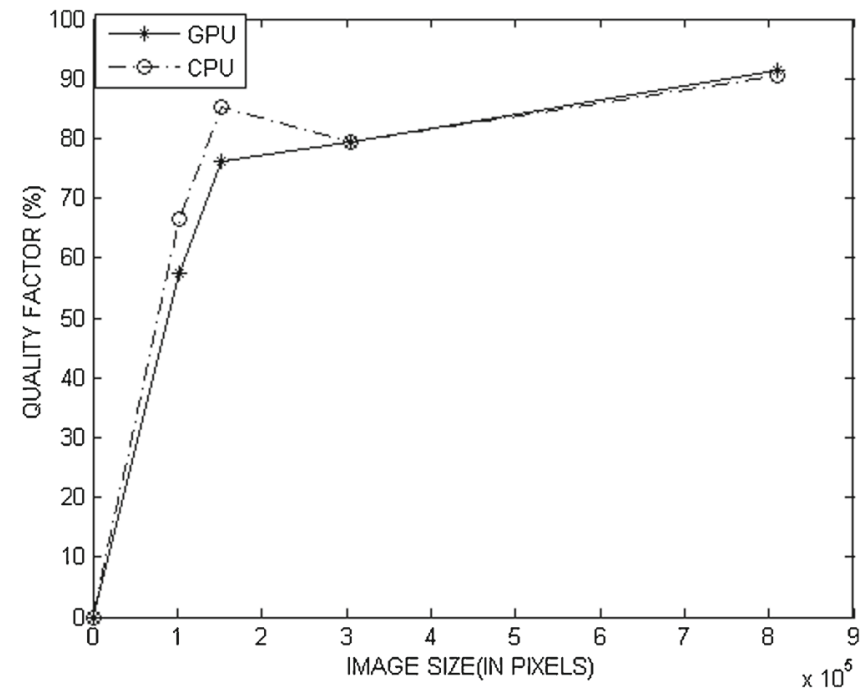

Figure 9. Image size vs. quality factor (\%).

The best result on the GPU is three times faster than the CPU version of the implementation using image size of $900 \times 900$ pixels. We have tried to reduce the computational complexity in time by implementing a part of the normalized cut algorithm on the GPU. This is the important contribution of this work.

The main advantage of using normalized cut method is that both inter-group and intra-group characteristics of the image are taken into account. Intra-group characteristics are incorporated into the similarity matrix which considers the similarity of pixels in the close neighbourhood. By considering both the local and global aspects of the image scene, normalized cuts works well in extracting roads. The algorithm is able, to an extent, to ignore noise, small surface changes, and weak edges. However, in our observation, the parameters in the normalized cut segmentation algorithm have to be tuned carefully and they are image scene dependent. The empirically chosen parameters could pose hindrances while tuning the algorithm for images with different geographical backgrounds.

Although the results are very promising, there is still room for improvements. Hence, one work around, as a future inclusion would be, to apply appropriate post-processing techniques to remove 
the false road segments that do not belong to the feature of interest.

\section{Acknowledgements}

The IKONOS image covering Hobart, Australia was provided by the courtesy of GeoEye. The authors would like to thank Dr H Honne Gowda from KSTA, Bangalore, India, for providing the QuickBird image. Also, the authors would like to thank the anonymous reviewers for their comments that helped in improving this paper.

\section{References}

Abramov A, Kulvicius T, Worgotter F and Dellen B 2011 Real-Time Image Segmentation on a GPU; In: Facing the Multicore-Challenge, pp. 131-142.

Acclereyes T 2011 Jacket: GPU computing without CUDA programming; www.accelereyes.com/content/collateral/ HighLevelGPUComputing.

Barzohar M and Cooper D B 1996 Automatic finding of main roads in aerial images by using geometric-stochastic models and estimation; Pattern Analysis and Machine Intelligence, IEEE Trans. 18(7) 707-721.

Baumgartner A, Steger C, Mayer H, Eckstein W and Ebner H 1999 Automatic road extraction based on multi-scale grouping and context; Photogramm. Eng. Remote Sens. 65(7) 777-785.

Bong D B L, Lai K C and Joseph A 2009 Automatic road network recognition and extraction for urban planning; Int. J. Appl. Sci. Eng. Technol. 5(1) 54-59.

Chung F R K 1997 Spectral Graph Theory; Regional Conference Series in Mathematics, Providence, RI: Amer. Math. Soc.

Geman D and Jedynak B 1996 An active testing model for tracking roads in satellite images; Pattern Analysis and Machine Intelligence, IEEE Trans. 18(1) 1-14.

Grote A and Heipke C 2008 Road extraction for the update of road databases in suburban areas; Int. Arch. Photogramm. Remote Sens. 37 563-568.

Grote A, Butenuth M and Heipke C 2007 Road extraction in suburban areas based on normalized cuts; Int. Arch. Photogramm. Remote Sens. 36(3) 51-56.

Gruen A and Li H 1997 Semi-automatic linear feature extraction by dynamic programming and LSB-snakes; Photogramm. Eng. Remote Sens. 63(8) 985-994.

Haralick R M, Sternberg S R and Zhuang X 1987 Image analysis using mathematical morphology; Pattern Analysis and Machine Intelligence, IEEE Trans. $9532-550$.

Huang T, Yang G and Tang G Y 1979 A fast twodimensional median filtering algorithm; Acoustics, Speech and Signal Processing, IEEE Trans. 27(1) 13-18.
Kong J, Dimitrov M, Yang Y, Liyanage J, Cao L, Staples J, Mantor M and Zhou H 2010 Accelerating MATLAB image processing toolbox functions on GPUs; GPGPU'10, Pittsburg, PA, USA, pp. 1-11.

Mayer H, Hinz S, Bacher U and Baltsavias E 2006 A test of automatic road extraction approaches; Int. Arch. Photogramm. Remote Sens. Spatial Infor. Sci. 36(3) 209-214.

Moreland K and Angel E 2003 The FFT on a GPU; In: Graphics Hardware, pp. 112-119. http://www.cs.unm. $\mathrm{edu} / \sim \mathrm{kmorel} /$ documents/fftgpu/.

Pan L, Gu L and Xu J 2008 Implementation of medical image segmentation in cuda, Proc. Int. Conf. on Technology and Applications in Biomedicine, pp. 82-85.

Park S R and Kim T 2001 Semi-automatic road extraction algorithm from IKONOS images using template matching; Proc. 22nd Asian Conference on Remote Sensing, pp. $1209-1213$.

Rubin G, Sager E V and Berger D H 2011 GPU Acceleration of SAR/ISAR Imaging Algorithms, http://www. accelereyes.com/examples/customer_papers_and_talks.

Senthilnath J, Rajeshwari M and Omkar S N 2009 Automatic road extraction using high resolution satellite image based on texture progressive analysis and normalized cut method; J. Indian Soc. Remote Sens. 37(3) 351-361.

Senthilnath J, Vikram Shenoy H, Rajendra Ritwik, Omkar S N, Mani V and Diwakar P G 2013 Integration of speckle de-noising and image segmentation using Synthetic Aperture Radar image for flood extent extraction; J. Earth Syst. Sci. 122(3) 559-572.

Seo N 2006 Normalized Cuts and Image Segmentation; Technical Report TR-ENEE731 Project.

Shi J and Malik J 2000 Normalized cuts and image segmentation; Pattern Analysis and Machine Intelligence, IEEE Trans 22(8) 888-905.

Valero S, Chanussot J, Benediktsson J A, Talbot H and Waske B 2010 Advanced directional mathematical morphology for the detection of the road network in very high resolution remote sensing images; Pattern Recognition Letters 31(10) 1120-1127.

Wendykier P and Nagy J G 2011 Image Processing on Modern CPUs and GPUs; In: Technical Report TR-2008-023, http://www.mathcs.emory.edu/ technical-reports/techrep-00148.pdf.

Wiedemann C, Heipke C, Mayer H and Jamet O 1998 Empirical evaluation of automatically extracted road axes; In: Empirical Evaluation Methods in Computer Vision (eds) Bowyer K J and Jonathon Phillips P, IEEE Computer Society Press, Silver Spring, MD, pp. 172187. Also in: Proc. 9th Australasian Remote Sensing Photogramm. Conf., The University of New South Wales, Sydney, Paper No. 239 (CD).

Yang Z, Zhu Y and Pu Y 2008 Parallel image processing based on CUDA; In: International Conference on Computer Science and Software Engineering 3 198-201.

Youn J and Bethel J S 2004 Adaptive snakes for urban road extraction; Int. Arch. Photogramm. Remote Sens. and Spatial Infor. Sci. 35(3) 465-470. 